\title{
Estudo da suscetibilidade ao escurecimento por radiação gama de quartzo róseo-leitoso da província pegmatítica da Borborema
}

\author{
Milena Ribas de Miranda ${ }^{1}$, Raysa Sthefany Gomes Gonzaga², Pedro Luiz Guzzo ${ }^{3 *}$, Sandra de \\ Brito Barreto ${ }^{4}$, Joan Carles Melgarejo ${ }^{5}$
}

\begin{abstract}
Resumo Este estudo considera as transformações induzidas pela radiação $\gamma$ sobre os defeitos estruturais em quartzo róseo e leitoso da Província Pegmatítica da Borborema (Paraíba e Rio Grande do Norte, Brasil). Amostras de coloração rósea e leitosa foram irradiadas com doses de raios $\gamma\left({ }^{60} \mathrm{Co}\right)$ entre 0,5 e $96 \mathrm{kGy}$. Defeitos pontuais relacionados às impurezas de $\mathrm{Al}, \mathrm{Ge}, \mathrm{Li}$ e grupos $\mathrm{OH}$ foram caracterizados por espectroscopias ultravioleta visível e infravermelha e ressonância paramagnética eletrônica, antes e após a irradiação. A espectrometria de massa com plasma indutivamente acoplado foi empregada para quantificar as concentrações de $\mathrm{Al}, \mathrm{Li}, \mathrm{Ge}, \mathrm{Fe}$, Ti e outras impurezas em fragmentos de diferentes tonalidades. Constatou-se que os defeitos $\left[\mathrm{AlO}_{4}\right]^{0},\left[\mathrm{AlO}_{4} / \mathrm{H}\right]^{0} \mathrm{e}\left[\mathrm{GeO}_{4} / \mathrm{Li}\right]^{0}$ são formados pela dissociação dos defeitos $\left[\mathrm{AlO}_{4} / \mathrm{Li}\right]^{0}$ e $[\mathrm{Li}-\mathrm{OH}]$, a partir de doses de $0,5 \mathrm{kGy}$. A diminuição do sinal da ressonância paramagnética eletrônica do centro $\left[\mathrm{GeO}_{4} / \mathrm{Li}\right]^{0}$ e a formação de centros E' ${ }_{1}$ perturbados por Ge para doses acima de $8 \mathrm{kGy}$ confirmou a grande mobilidade dos íons $\mathrm{Li}^{+}$pela estrutura do quartzo. $\mathrm{O}$ aumento da população de defeitos $\left[\mathrm{AlO}_{4}\right]^{0} \mathrm{e}$ o consequente escurecimento das amostras foi semelhante para ambos os tipos de quartzo. Análises preliminares por microscopia eletrônica mostraram a presença de fibras nanométricas apenas no resíduo da digestão ácida de amostras róseas, indicando que a coloração rósea do quartzo da Província Pegmatítica da Borborema está provavelmente relacionada às inclusões de dumortierita.
\end{abstract}

Palavras-chave: quartzo natural; pegmatito granítico; radiação ionizante; espectroscopia; defeitos pontuais.

\begin{abstract}
Impurity-related point defects and gamma-radiation response of massive quartz from the Borborema pegmatite province, in Brazil. This work has investigated the changes induced by $\gamma$-radiation on impurity-related point defects in massive rose quartz from one deposit located at The Borborema Pegmatite Province (Northeast Region, in Brazil). Samples extracted from rose and colorless (milky) quartz blocks were irradiated with doses of ${ }^{60} \mathrm{Co}$, from 0.5 to $96 \mathrm{kGy}$. Point defects related to $\mathrm{Al}, \mathrm{Ge}, \mathrm{Li}$ and $\mathrm{OH}$ were measured by optical, infrared, and electron paramagnetic resonance spectroscopy, prior and after irradiation. The contents of $\mathrm{Al}, \mathrm{Li}, \mathrm{Ge}, \mathrm{Fe}, \mathrm{Ti}$ and other impurities were measured by inductively-coupled plasma mass spectrometry in quartz fragments exhibiting rose, pale-rose, and milky colorations. It was found that $\left[\mathrm{AlO}_{4}\right]^{0},\left[\mathrm{AlO}_{4} / \mathrm{H}\right]^{0}$ and $\left[\mathrm{GeO}_{4} / \mathrm{Li}\right]^{0}$ were generated by the dissociation of $\left[\mathrm{AlO}_{4} / \mathrm{Li}\right]^{0}$ and $[\mathrm{Li}-\mathrm{OH}]$ centers with doses as lower as $0.5 \mathrm{kGy}$. Above $8 \mathrm{kGy}$, the electron paramagnetic resonance signal related to $\left[\mathrm{GeO}_{4} / \mathrm{Li}\right]^{0} \mathrm{decreases}$ due to the intense mobility of $\mathrm{Li}$ species throughout the quartz lattice, giving rise to $\mathrm{E}_{1}$, centers perturbed by Ge. The increase in $\left[\mathrm{AlO}_{4}\right]^{0}$ content with $\gamma$ doses and the consequent rise in the intensity of smoky color were similar for both rose and colorless quartz. Scanning electron microscopy carried out in insoluble residues obtained after chemical dissolution of each type of quartz revealed the presence of nanometric fibers only in rose specimens. These results suggested that the cause of rose color in massive quartz from Borborema Pegmatite Province is probably related to the presence of dumortierite inclusions.
\end{abstract}

Keywords: natural quartz; granitic pegmatite; ionizing radiation; spectroscopy; point defects.

\section{INTRODUÇÃOO Os pegmatitos da Província} da Borborema estão concentrados em uma área de aproximadamente $75 \times 150 \mathrm{~km}^{2}$, no Sudeste da faixa de dobramento do Seridó, localizada nos estados da
Paraíba e do Rio Grande do Norte. Aproximadamente $80 \%$, dentre os 750 corpos até então conhecidos, são pegmatitos graníticos, encaixados em granada-cordierita e/ou silimanita-biotita-xistos da Formação

${ }^{1}$ Programa de Pós-Graduação em Engenharia Mineral da Universidade Federal de Pernambuco - UFPE, Recife (PE), Brasil. E-mail: milaribas@hotmail.com

${ }^{2}$ Departamento de Engenharia de Minas da Universidade Federal de Pernambuco - UFPE, Recife (PE), Brasil. E-mail: raysagonzaga@hotmail.com

${ }^{3}$ Departamento de Engenharia de Minas da Universidade Federal de Pernambuco - UFPE, Recife (PE), Brasil. E-mail: pguzzo@ufpe.br ${ }^{4}$ Departamento de Geologia da Universidade Federal de Pernambuco - UFPE, Recife (PE), Brasil. E-mail: sandrabrito@smart.net.br ${ }^{5}$ Departamento de Cristalografia, Mineralogia e Depósitos Minerais da Universidade de Barcelona, E-08028, Barcelona, Espanha.

E-mail: joan.carles.melgarejo.draper@ub.edu

*Autor correspondente 
Seridó. A partir da Segunda Guerra Mundial, a Província Pegmatítica da Borborema (PPB) tornouse mundialmente conhecida pelas reservas de nióbio-tantalatos, scheelita e minerais gemas (turmalinas e berilo). Atualmente, além de turmalinas de rara beleza, os pegmatitos da Borborema são fontes de matérias-primas para as indústrias cerâmicas da região e do país, notadamente feldspatos, muscovita, quartzo e caulim (Da Silva et al. 1995, Beurlen et al. 2009).

Há décadas, os pegmatitos da PPB são explorados por meio de lavras artesanais ou semimecanizadas em busca de gemas, minerais exóticos e nióbio-tantalatos (Luz et al. 2003). Esta prática extrativista causou e vem causando, nas áreas circunvizinhas às banquetas, pilhas de rejeitos, compostas principalmente por quartzo leitoso de qualidade óptica inferior, feldspatos e micas. Aplicações inovadoras para tais rejeitos minerais são, portanto, altamente desejáveis tanto do ponto de vista econômico quanto do ambiental. Por exemplo, o escurecimento de quartzo leitoso, por meio do bombardeamento com raios $\gamma$, poderia ser uma alternativa para $o$ aproveitamento deste insumo ao artesanato mineral. $\mathrm{O}$ uso da radiação ionizante para o desenvolvimento de novas cores e tonalidades de minerais gemas ou cristais cultivados em laboratório é prática recorrente da indústria joalheira; sobretudo, quando a natureza dos agentes responsáveis pelas colorações desejadas é conhecida (Koivula 1980, Balistky et al. 1998, Lameiras et al. 2006).

Como se sabe, a coloração da maioria das variedades de quartzo atribui-se à presença de defeitos pontuais resultantes da incorporação de impurezas substitucionais e/ou intersticiais e da disponibilidade de radiação ionizante no local de crescimento dos cristais. O melhor caso resolvido é o da coloração esfumaçada (smoky) de cristais euédricos, resultantes da presença de defeitos $\left[\mathrm{AlO}_{4}\right]^{0}$ formados por um buraco aprisionado em um dos orbitais $p$ de um dos íons oxigênio do tetraedro, no qual o íon $\mathrm{Al}^{3+}$ substituiu o $\mathrm{Si}^{4+}$ (O’Brien 1954, Nuttal \& Weil 1981).

Para a cor violeta da ametista, o modelo mais aceito considera a presença de impurezas férricas no estado tetravalente $\left(\mathrm{Fe}^{4+}\right)$, com coordenação tetraédrica resultante da dissociação de centros do tipo $\left[\mathrm{FeO}_{4} / \mathrm{Li}\right]^{0}$ pela radiação ionizante (Lehmann \& More 1966, Cox 1977, Rossman 1994). A cor amarela do quartzo citrino também é atribuída às impurezas de $\mathrm{Fe}^{3+}$; porém, na forma de precipitados $\mathrm{Fe}_{2} \mathrm{O}_{3}$ decorrentes do tratamento térmico da ametista (Rossman 1994). Desde o trabalho inicial de Holden (1924), várias hipóteses foram propostas para explicar a natureza da coloração rósea do quartzo. Dentre elas, Smith et al. (1978) propuseram um modelo de transferência de cargas entre os níveis de valência do ferro e titânio $\left(\mathrm{Fe}^{2+}+\mathrm{Ti}^{4+} \rightarrow\right.$ $\mathrm{Fe}^{3+}+\mathrm{Ti}^{3+}$ ) para explicar o surgimento da banda de absorção a aproximadamente $500 \mathrm{~nm}$, presente nos espectros de cristais róseos. Posteriormente, analisando o sinal da ressonância paramagnética eletrônica (RPE) de um exemplar monocristalino de quartzo róseo euédrico, Machmeyer \& Lehmann (1983) propuseram que a coloração rósea seria consequência de um centro de buraco, relacionado às impurezas substitucionais de $\mathrm{Al} \mathrm{e} \mathrm{P}$ induzido por radiação ionizante. Após análise de vários trabalhos, Rossman (1994) relatou que os agentes responsáveis pela coloração rósea de blocos extraídos de núcleos pegmatíticos não são os mesmos que atuam nos cristais euédricos, ocorrendo menos frequentemente. Mais recentemente, a coloração rósea do quartzo foi atribuída à transferência de carga entre íons $\mathrm{Fe}^{2+}$ e $\mathrm{Ti}^{4+}$, os quais supostamente ocorrem como impurezas em inclusões de dumortierita $\left(\mathrm{Al}_{7}\left(\mathrm{BO}_{3}\right)\left(\mathrm{SiO}_{4}\right) \mathrm{O}_{3}\right)($ Goreva et al. 2001, $\mathrm{Ma}$ et al. 2002, Kibar et al. 2007). Anteriormente, fibras submicroscópicas de dumortierita com coloração rósea foram observadas em resíduos insolúveis de quartzo róseo, por Applin \& Hicks (1987).

Neste contexto, por meio de técnicas espectroscópicas complementares e pela análise da concentração de impurezas, o objetivo deste estudo foi investigar as modificações na coloração de quartzo de tonalidades rósea e leitosa mediante a exposição de diferentes amostras a doses de radiação $\gamma$ de até $96 \mathrm{kGy}$. O escurecimento gradativo de cada tipo de quartzo foi analisado em função das transformações ocorridas na população de defeitos relacionados às impurezas de $\mathrm{Al}, \mathrm{Ge}, \mathrm{Li}$ e grupos $\mathrm{OH}$. Por meio de análises preliminares de microscopia eletrônica de varredura, este trabalho também teve o objetivo de contribuir para o esclarecimento da natureza da coloração rósea do quartzo oriundo dos pegmatitos graníticos da PPB.

MATERIAIS E MÉTODOS Blocos de quartzo róseo-leitosos, com alta densidade de fraturas e pequena transparência, foram extraídos do núcleo 
do Pegmatito Taboa, que é localizado no município de Carnaúba dos Dantas, Rio Grande do Norte. Essencialmente, o núcleo deste pegmatito é constituído dos quartzos leitoso e róseo, com distribuição aleatória e passagem transicional entre as colorações (Barreto et al. 2009). Em função da distribuição e intensidade da coloração rósea, foram definidos três pontos de coleta, segundo um perfil transversal ao núcleo, no sentido E-W, de onde foram extraídos blocos de quartzos leitoso (desprovido de qualquer sinal de coloração rósea), róseopálido e de coloração rósea mais acentuada. A Fig. 1 apresenta imagens de dois blocos coletados.

Dez amostras dos blocos de tonalidade rósea (acentuada) e leitosa foram cortadas na forma de placas, com superfícies de aproximadamente $12 \times 12 \mathrm{~mm}^{2}$ utilizando uma serra de disco diamantado. As amostras róseas e leitosas foram cortadas com espessuras de 3,5 e 3,0 mm, respectivamente. As superfícies das amostras foram lapidadas sobre placa de vidro com grãos abrasivos de alumina $\left(\mathrm{Al}_{2} \mathrm{O}_{3}\right)$, de granulometria decrescente $(75,38,20$, 6 e $2 \mu \mathrm{m})$. Em seguida, utilizando uma lapidadora de disco plano recoberto com tecido sintético, as superfícies foram polidas opticamente com suspensão de $\mathrm{Al}_{2} \mathrm{O}_{3}$ de $1 \mu \mathrm{m}$. A espessura final das amostras róseas e leitosas foi de aproximadamente 3,0 e $2,4 \mathrm{~mm}$, respectivamente. A irradiação das amostras foi realizada com raios $\gamma$ de uma fonte gammacell de ${ }^{60} \mathrm{Co}$, modelo 220 Excell MDS Nordion, com taxa de dose de $6,77 \mathrm{kGy} / \mathrm{h}$. As doses de radiação variaram de 0,5 a $96 \mathrm{kGy}$. Algumas amostras são mostradas na Fig. 1.

Os espectros de absorção nas faixas ultravioleta visível (UV-Vis) e infravermelho (IV) médio foram realizados à temperatura ambiente antes e depois das amostras serem irradiadas. Os espectros UV-Vis foram obtidos com um espectrômetro de feixe duplo, modelo Lambda 35 da Perkin Elmer, com varredura de $120 \mathrm{~nm} / \mathrm{min}$., feixe de luz nãopolarizado, fenda de $4 \mathrm{~nm}$ e comprimento de onda variando de 1.100 a $190 \mathrm{~nm}$. Por sua vez, os espectros IV foram obtidos com um espectrômetro ABB Bomem FTLA 2000, com luz não polarizada e resolução melhor que $4 \mathrm{~cm}^{-1}$. Cada espectro foi obtido após 50 varreduras entre 500 e $6.000 \mathrm{~cm}^{-1}$. Em ambas as medidas, uma janela com diâmetro de $3 \mathrm{~mm}$ foi utilizada para delimitar o feixe de luz sobre a amostra e garantir que os espectros fossem obtidos nas regiões de maior transparência.
A intensidade das bandas de absorção nos espectros UV-Vis foi determinada pela equação de Beer-Lambert (1), cuja forma geral é:

$$
\alpha=\frac{1}{t}\left(A-A_{0}\right)
$$

onde:

$t$ é a espessura da amostra $(\mathrm{cm})$,

$A$ é a absorbância na posição de máxima absorção, antes $\left(A_{0}\right)$ e após $(A)$ irradiação,

$\alpha$ é o coeficiente de absorção decimal $\left(\mathrm{cm}^{-1}\right)$.

A Fig. 2a apresenta espectros característicos de amostras com tonalidades rósea e leitosa, obtidos antes (condição natural) e após a irradiação. Nos espectros UV-Vis, determinou-se as intensidades das bandas relacionadas ao centro $\left[\mathrm{AlO}_{4}\right]^{0}$ (460 e $620 \mathrm{~nm}$ ). Por sua vez, nos espectros IV foram medidas as bandas relacionadas aos centros $\left[\mathrm{H}_{4} \mathrm{O}_{4}\right]^{0}$, [Li$\mathrm{OH}]$ e $\left[\mathrm{AlO}_{4} / \mathrm{H}\right]^{0}$ que ocorrem a aproximadamente $3.595,3.484$ e $3.380 \mathrm{~cm}^{-1}$, respectivamente (Kats 1962, Bahadur 1989, Guzzo et al. 1997).

Nos espectros IV, determinou-se a absorbância das bandas na condição irradiada, uma vez que não se dispunha dos espectros IV na condição natural. Para cada banda, as transmitâncias máxima e mínima foram determinadas pelo método da densidade de linha de base (Zichy 1972). A partir daí, utilizou-se a equação (1) para a determinação de $\alpha$,

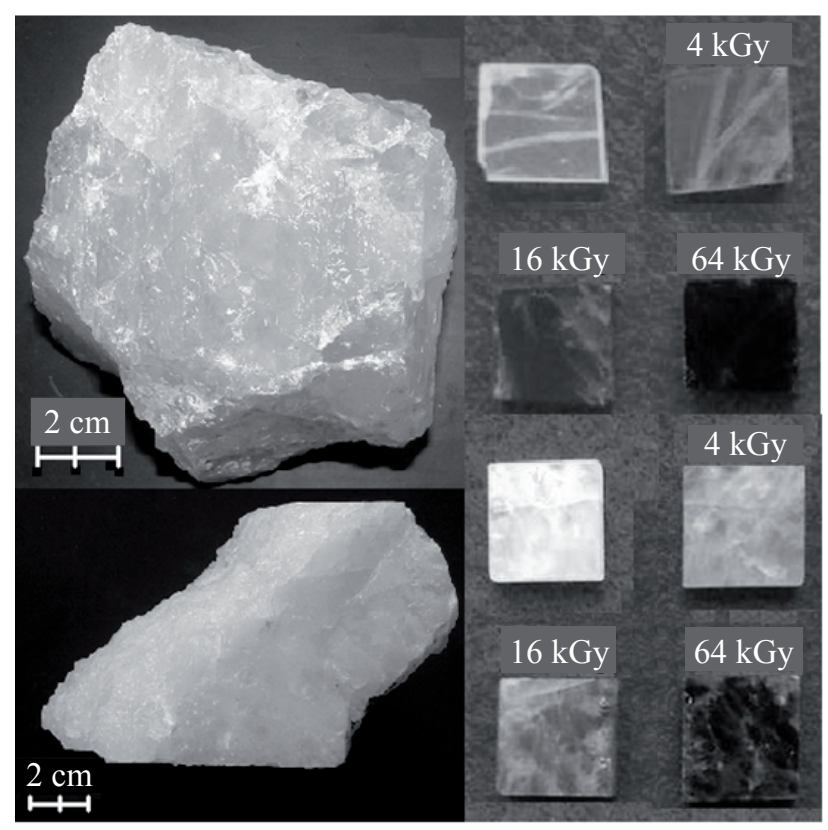

Figura 1-Blocos de quartzo róseo e leitoso coletados do núcleo do Pegmatito Taboa (RN) e amostras irradiadas com raios $\gamma\left({ }^{60} \mathrm{Co}\right)$. 
assumindo $A_{0}=0$. Nos espectros da Fig. 2B, notou-se que as bandas IV citadas se encontram superpostas a uma banda larga isotrópica; consequência das vibrações de estiramento dos Grupos $\mathrm{OH}$ e das moléculas de água aprisionadas na estrutura do quartzo (Aines \& Rossman 1984). As bandas de absorção a 3.300 e $3.200 \mathrm{~cm}^{-1}$ são atribuídas aos harmônicos das vibrações Si-O (Kats 1962), as quais foram discretamente observadas nos espectros de quartzo leitoso (Fig. 2C) devido à baixa transmitância dessas amostras, decorrente da grande densidade de fraturas.

Para as medidas por espectroscopia de RPE, parte de cada placa foi cominuída em almofariz de ágata, com a finalidade de produzir alíquotas de aproximadamente $60 \mathrm{mg}$ com granulometria entre 75 e $150 \mu \mathrm{m}$. As alíquotas foram colocadas em tubos de sílica vítrea de alta pureza (diâmetro interno igual a $2 \mathrm{~mm}$ ), e as medidas foram realizadas com um espectrômetro Bruker $\mathrm{EMX}^{+}$, operando na banda $\mathrm{X}(\sim 9,83 \mathrm{GHz})$, à temperatura ambiente $\left(\sim 23^{\circ} \mathrm{C}\right)$.
Após várias varreduras para localização dos sinais, os espectros foram registrados com potências entre 0,5 e $20 \mathrm{~mW}$ na faixa de 3.440 e $3.560 \mathrm{G}$. As demais condições de medida foram: amplitude de modulação: $1 \mathrm{G}$; frequência de modulação: $100 \mathrm{kHz}$; constante de tempo: $10,24 \mathrm{~ms}$; tempo de conversão: 20 ms e ganho: $1 \times 10^{4}$. Os fatores $\mathrm{g}$ dos sinais mais intensos foram identificados e suas intensidades foram medidas para amostras irradiadas e na condição natural. A Fig. 2D apresenta espectros de RPE característicos de quartzo róseo. Neles, foram identificados os sinais relacionados aos centros $\left[\mathrm{GeO}_{4} /\right.$ $\mathrm{Li}]^{0}$ e $\mathrm{E}_{1}$, perturbado por $\mathrm{Ge}$, aqui representado por $\left[\mathrm{E}_{1}{ }_{1}-\mathrm{Ge}\right]^{0}$ (Mackey 1963, Weil 1984). As amostras de quartzo leitoso apresentaram sinais RPE semelhantes ao do róseo.

Para a determinação das concentrações de impurezas, fragmentos de cada quartzo (róseo, róseo-pálido e leitoso) foram cominuídos em almofariz de ágata, e alíquotas de $3 \mathrm{~g}$ foram encaminhadas ao Laboratório de Química dos Serviços Técnico-
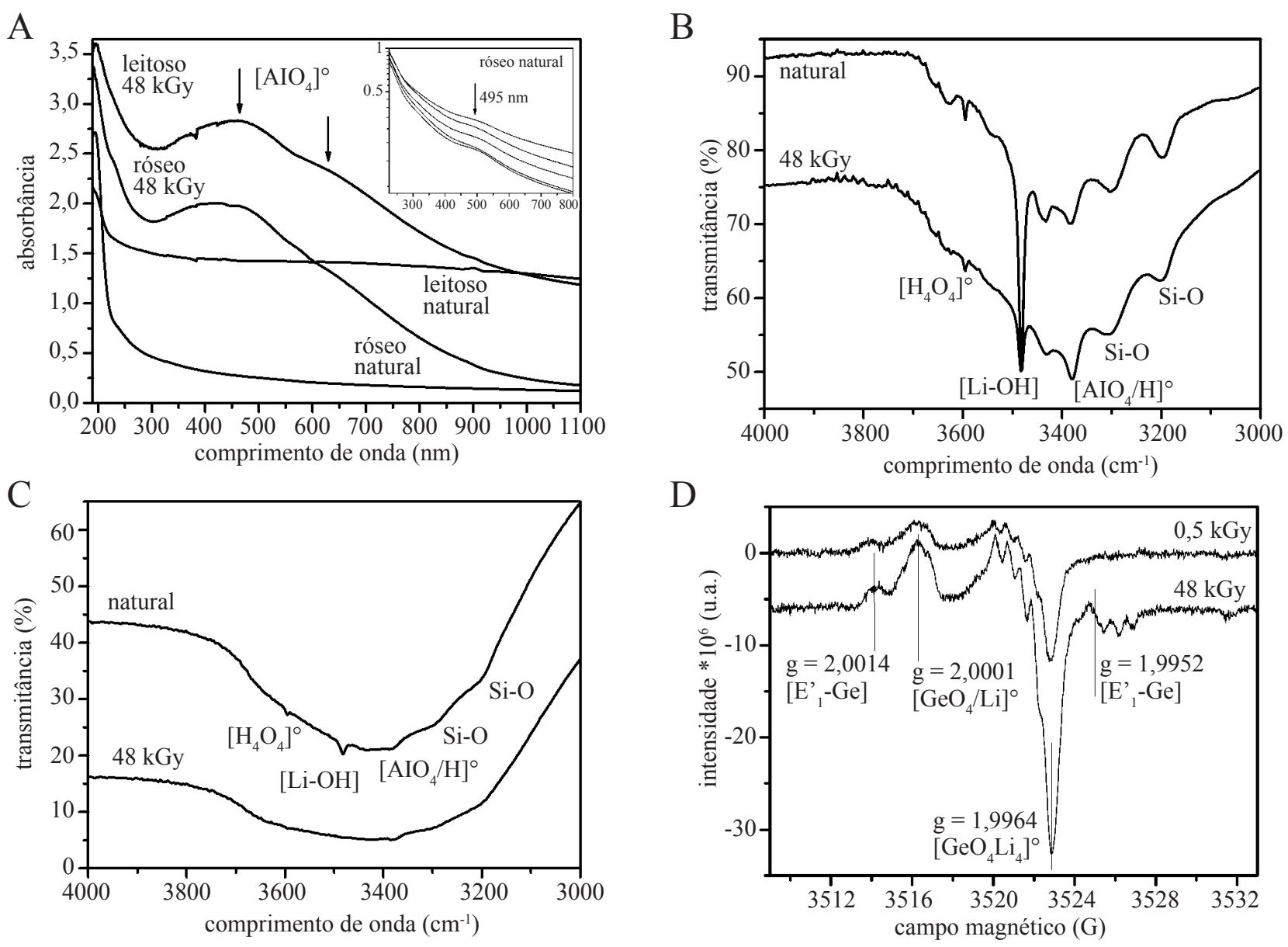

Figura 2 - Espectros UV-Vis, IV e RPE característicos de quartzo róseo e leitoso nas condições natural e irradiados com raios $\gamma\left({ }^{60} \mathrm{Co}\right)$ : (A) UV-Vis; (B) IV-róseo; (C) IV-leitoso; (D) RPE-róseo. 
Científicos da Universidade de Barcelona, onde foram submetidas aos procedimentos de ataque ácido e abertura de amostra. Em seguida, as concentrações de Al, P, Ge, Fe, Ti, Cr, Ni, Li, Na, K, Rb, Mg, Ca, $\mathrm{Sr}$ e B foram determinadas por espectrometria de massa com plasma indutivamente acoplado (ICPMS), utilizando o espectrômetro ICM-MS 7500ce da Agilent Technologies.

Visando examinar a presença de inclusões de dumortierita, aproximadamente $17 \mathrm{~g}$ de fragmentos de tamanho entre 1,5 e 5,0 $\mathrm{mm}$ dos blocos róseo e leitoso foram submetidos separadamente à digestão ácida com ácido fluorídrico (HF), durante 48 horas, à aproximadamente $70{ }^{\circ} \mathrm{C}$. O procedimento de digestão e limpeza do resíduo foi semelhante àquele realizado por Goreva et al. (2001). $\mathrm{O}$ resíduo da digestão, verificado apenas para o quartzo róseo $(\sim 6,0 \mathrm{mg}$ de material rosado), foi analisado por microscopia eletrônica de varredura, com um microscópio Quanta-200-FEG da FEI Company, equipado com detectores de elétrons secundários (SE), elétrons retroespalhados (BS) e espectrômetro de dispersão de energia (EDS), no Laboratório de Microscopia e Microanálise (CETENE/MCT, Recife, Pernambuco).

RESULTADOS A Fig. 3 apresenta as intensidades das bandas de absorção medidas pelas espectroscopias UV-Vis, IV e RPE em função da dose de radiação à qual as amostras de quartzo róseo $\mathrm{e}$ leitoso foram expostas. Na Fig. 3A, o aumento da banda de absorção a $460 \mathrm{~nm}$ indica aquele da população de defeitos $\left[\mathrm{AlO}_{4}\right]^{0}$, responsável pelo aparecimento da coloração esfumaçada em ambos os tipos de quartzo. Esta banda aparece com menor dose de radiação no quartzo róseo ( $2 \mathrm{kGy}$ ) do que no leitoso (8 kGy). Ainda, observa-se uma aparente saturação desta banda para o quartzo leitoso, ou seja, sua intensidade não aumenta para doses acima de $48 \mathrm{kGy}$. Acredita-se que esta saturação seja em parte atribuída à grande densidade de fraturas no quartzo leitoso. Tais fraturas causam o espalhamento da luz, elevando o sinal de fundo (aumento da absorbância na faixa de varredura, como pode ser observado na
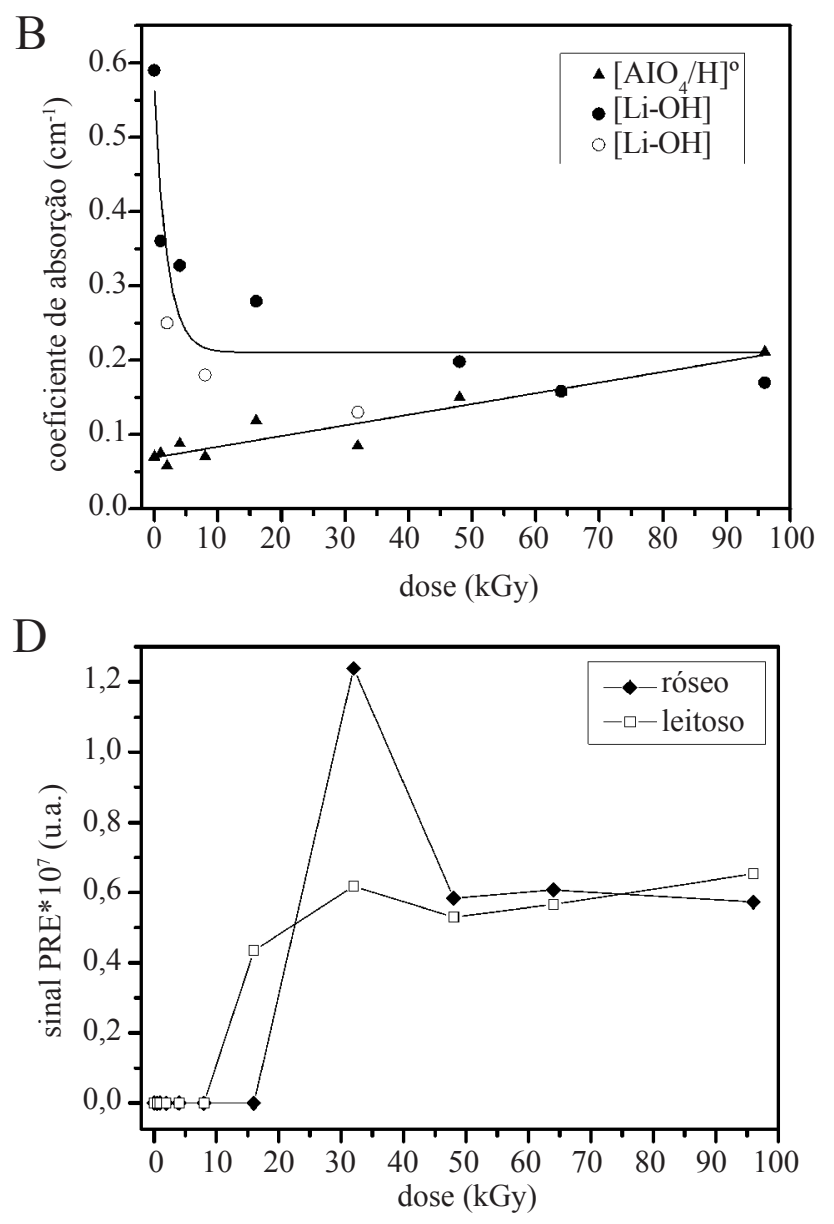

Figura 3 - Intensidade das bandas de absorção de defeitos pontuais em quartzo- róseo e leitoso em função da dose de radiação $\gamma\left({ }^{60} \mathrm{Co}\right)$ : (A) $\left[\mathrm{AlO}_{4}\right]^{\circ}$; (B) $[\mathrm{AlO} / \mathrm{H}]^{0}$ e $[\mathrm{Li}-\mathrm{OH}]$; (C) $[\mathrm{GeO} / \mathrm{Li}]^{0}$; (D) $\left[\mathrm{E}_{1}{ }_{1}-\mathrm{Ge}\right]^{0}$. 
Fig. 2(A)) e dificultando a estimativa dos coeficientes de absorção.

A densidade das fraturas também dificultou a medida das bandas IV no quartzo leitoso. Por este motivo, na Fig. 3B, são apresentadas apenas as intensidades das bandas IV do quartzo róseo. Nela, observou-se uma tendência de crescimento linear da banda associada ao defeito $\left[\mathrm{AlO}_{4} / \mathrm{H}\right]^{0}$ em função da dose. No caso do defeito [Li-OH], observou-se que a intensidade da banda a $3.484 \mathrm{~cm}^{-1}$ decresce de forma acentuada até $8 \mathrm{kGy}$ para, em seguida, estabilizar-se em torno de um valor muito inferior àquele encontrado para amostras na condição natural. A espectrosopia IV também mostrou que a banda a $3.595 \mathrm{~cm}^{-1}$ não foi afetada de forma significativa pela radiação $\gamma$, sugerindo que a população de defeitos $\left[\mathrm{H}_{4} \mathrm{O}_{4}\right]^{0}$ permaneceu aproximadamente constante. $\mathrm{O}$ modelo proposto para descrever o defeito $\left[\mathrm{H}_{4} \mathrm{O}_{4}\right]^{0}$ corresponde à substituição do íon $\mathrm{Si}^{4+}$ por quatro prótons $\mathrm{H}^{+}$(Nuttall \& Weil 1980, Lipson \& Kahan 1985).

As Figs. 3C e 3D apresentam o comportamento dos sinais RPE dos centros $\left[\mathrm{GeO}_{4} / \mathrm{Li}\right]^{0}$ e $\left[\mathrm{E}_{1}{ }_{1}-\mathrm{Ge}\right]^{0}$ em função da dose de radiação $\gamma$. Em ambos os tipos de quartzo, estes sinais não foram observados em amostras na condição natural. $\mathrm{Na}$ Fig. 3c, observou-se que o sinal associado ao defeito $\left[\mathrm{GeO}_{4} / \mathrm{Li}\right]^{0}$ aumenta rapidamente até $4 \mathrm{kGy}$ e depois começa a diminuir de forma acentuada até $32 \mathrm{kGy}$ e de forma mais lenta para maiores doses. A análise de ambos os sinais mostra que o defeito $\left[\mathrm{E}_{1}{ }_{1}-\mathrm{Ge}\right]^{0}$ aparece quando a intensidade do sinal do defeito $\left[\mathrm{GeO}_{4} / \mathrm{Li}\right]^{0}$ está diminuindo, ou seja, a partir de $8 \mathrm{kGy}$ para o quartzo leitoso e $16 \mathrm{kGy}$ para o róseo. Também observa-se que a redução do sinal do centro $\left[\mathrm{GeO}_{4} / \mathrm{Li}\right]^{0}$ no quartzo leitoso ocorre a uma taxa mais acentuada do que no róseo.

A Tab. 1 apresenta as concentrações dos elementos menores e traços, determinadas pela espectrometria de massa com plasma indutivamente acoplado (ICP-MS, nas siglas em inglês) nas três variedades de quartzo. Para os estudos dos possíveis defeitos presentes na estrutura cristalina, estes elementos são considerados como impurezas. As impurezas dominantes são $\mathrm{Al}$ e $\mathrm{Li}$ e, em menor concentração, Ti e Ge. Tais elementos são comumente encontrados na estrutura do quartzo natural, na forma de impurezas substitucionais ou intersticiais (Götze et al. 2004, Larsen et al. 2004, Beurlen et al. 2009b). Não foi detectada a presença de $\mathrm{P}, \mathrm{Mg}$, $\mathrm{Ca}$ e Sr. A grande concentração de $\mathrm{Cr}$, Fe e Ni medida apenas na variedade rósea pode ser explicada pela presença de inclusões sólidas microscópicas, de morfologia arredondada, observadas no resíduo da digestão ácida. Por sua vez, o Na, em concentração significativa na amostra de quartzo leitoso, pode ser proveniente de inclusões fluidas e, eventualmente, ocorrer como compensador de carga dos centros de Al (Iwasaki et al. 1991).

A Fig. 4 apresenta os resultados preliminares obtidos com a caracterização do resíduo insolúvel da digestão ácida do quartzo róseo. A imagem obtida com elétrons retroespalhados apresenta uma

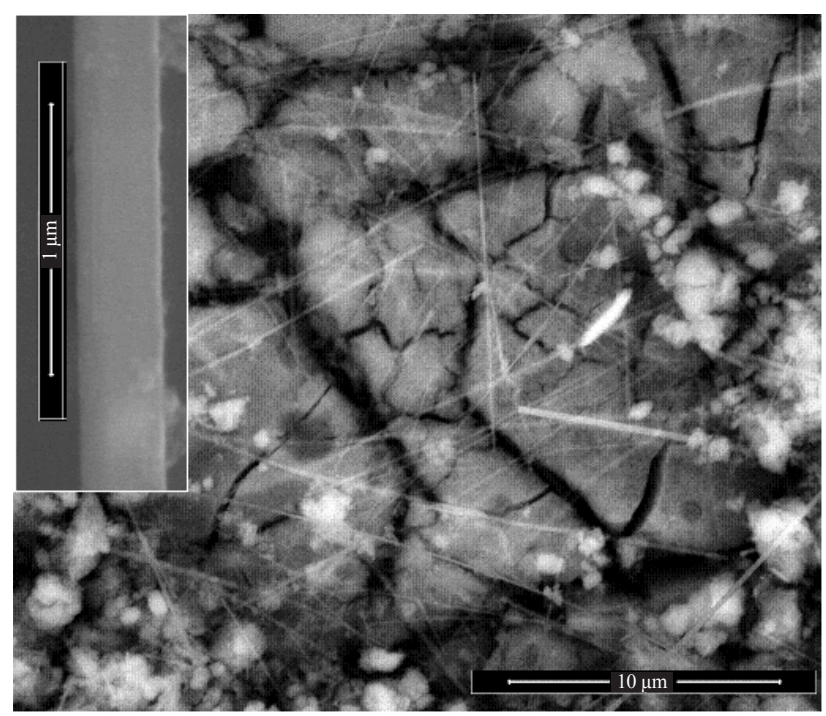

Figura 4 - Micrografia (elétrons retroespalhados e secundários) do resíduo da digestão ácida de fragmentos do quartzo róseo.

Tabela 1 - Impurezas medidas por espectrometria ICP-MS em três variedades de quartzo róseo-leitoso do Pegmatito Taboa (PPB)

\begin{tabular}{|c|c|c|c|c|c|c|c|c|c|c|c|}
\hline \multirow{2}{*}{ Variedade } & \multicolumn{11}{|c|}{ Concentração de impurezas (N/10 $\mathrm{Si})$} \\
\hline & $\mathrm{Al}$ & $\mathrm{Ti}$ & $\mathrm{Cr}$ & $\mathrm{Fe}$ & $\mathrm{Ni}$ & $\mathrm{Ge}$ & $\mathrm{Li}$ & $\mathrm{Na}$ & $\mathrm{K}$ & $\mathrm{Rb}$ & $\mathrm{B}$ \\
\hline Limite de detecção & 4,5 & 1,3 & 4,6 & 2,2 & 4,1 & 0,1 & 0,3 & 52,3 & 15,4 & 0,03 & 5,6 \\
\hline Róseo & 600,4 & 20,5 & $1.929,7$ & $6.873,5$ & 773,8 & 1,6 & 376,8 & n.d. & n.d. & n.d. & n.d. \\
\hline Róseo-pálido & 688,5 & 12,2 & n.d. & n.d. & 2,4 & n.d. & 237,4 & 64,0 & 23,5 & 0,05 & 6,6 \\
\hline Leitoso & 400,5 & 3,6 & n.d. & n.d. & 2,7 & n.d. & 98,0 & 215,0 & n.d. & 0,06 & n.d. \\
\hline
\end{tabular}

n.d.: não detectado. 
grande quantidade de fibras finas sobre um aglomerado de material aparentemente amorfo. A análise por EDS em algumas regiões desse material revelou a presença de $\mathrm{O}, \mathrm{Na}, \mathrm{Al}, \mathrm{Si}, \mathrm{P}, \mathrm{S}, \mathrm{K}, \mathrm{Ca}, \mathrm{Ti}, \mathrm{Cr}$ e Fe. Em particular, a presença de $\mathrm{Ti}, \mathrm{Cr}$ e $\mathrm{Fe}$ se mostrou mais concentrada em algumas regiões do que em outras, em decorrência das microinclusões arredondadas. As imagens de elétrons secundários sugerem que as fibras sejam inclusões cristalinas. A morfologia de uma fibra isolada, com aproximadamente 300 nm de espessura, é mostrada no detalhe da Fig. 4. O sinal EDS coletado nesta fibra apenas revelou a presença de $\mathrm{O}, \mathrm{Al}$ e Si.

DISCUSSÃo Os resultados deste estudo mostram que, na condição natural, os defeitos pontuais associados às impurezas substitucionais de $\mathrm{Al}$ e Ge na estrutura das variedades rósea e leitosa do Pegmatito Taboa são os centros $\left[\mathrm{AlO}_{4} / \mathrm{Li}\right]^{0}$, $\left[\mathrm{AlO}_{4} / \mathrm{H}\right]^{0}$ e $\left[\mathrm{GeO}_{4}\right]^{0}$. Essa constatação está baseada em trabalhos anteriores, os quais, usando técnicas complementares, identificaram a presença desses centros em quartzos hialino e sintético não irradiados (Mackey 1963, Halliburton et al. 1981, Martin 1984, Bahadur 1989, Guzzo et al. 1997, Götze et al. 2004). Muito embora os espectros UVVis tenham indicado a presença de bandas relacionadas ao defeito $\left[\mathrm{AlO}_{4}\right]^{0}$ apenas para doses iguais ou maiores que $2 \mathrm{kGy}$, acredita-se que a dissociação dos centros $\left[\mathrm{AlO}_{4} / \mathrm{Li}\right]^{0}$ e a consequente formação dos centros $\left[\mathrm{AlO}_{4}\right]^{0},\left[\mathrm{AlO}_{4} / \mathrm{H}\right]^{0}$ e $\left[\mathrm{GeO}_{4} / \mathrm{Li}\right]^{0}$ ocorram a partir de $0,5 \mathrm{kGy}$. Isto porque a espectroscopia RPE, utilizada para acompanhar a formação dos centros $\left[\mathrm{GeO}_{4} / \mathrm{Li}\right]^{0}$, é mais sensível do que a UV-Vis, utilizada para acompanhar os centros $\left[\mathrm{AlO}_{4}\right]^{0}$. Além disso, a redução da banda IV relacionada ao defeito [Li-OH] foi observada após a dose de 0,5 kGy, indicando a fácil mobilidade dos íons $\mathrm{Li}^{+}$neste quartzo. Outra evidência da mobilidade dos íons $\mathrm{Li}^{+}$é a gradativa dissociação dos defeitos $\left[\mathrm{GeO}_{4} / \mathrm{Li}\right]^{0}$, seguida da formação dos centros $\left[\mathrm{E}_{1}{ }_{1}-\mathrm{Ge}\right]^{0}$ para doses iguais ou maiores que $8 \mathrm{kGy}$.

Cabe analisar o fato de não terem sido observados centros E' " "puros" nos quartzos róseo e leitoso, antes e após a irradiação. O modelo aceito para o centro E' é aquele de um elétron aprisionado no orbital $s p^{3}$ de um íon $\mathrm{Si}^{4+}$, que se desloca para uma vacância de oxigênio. A ausência desses centros em quartzo irradiado à temperatura ambiente também foi constatada em cristais sintéticos e naturais de outras procedências (Jani et al. 1983, Götze et al. 2004). Segundo Jani et al. (1983), a produção de centros E', ocorre preferencialmente em cristais em que a compensação de carga dos centros de $\mathrm{Al}$ seja feita por íons alcalinos e que sejam, simultaneamente, irradiados à temperatura ambiente e tratados termicamente a $300{ }^{\circ} \mathrm{C}$. Como os centros de $\mathrm{Al}$ dos quartzos róseo e leitoso do Pegmatito Taboa são compensados predominantemente por íons $\mathrm{Li}^{+}$, a ausência de centros E', deve-se ao fato dos mesmos não terem sido tratados termicamente após a irradiação. Assim, concluiu-se que a formação dos centros $\left[\mathrm{E}_{1}{ }_{1}-\mathrm{Ge}\right]^{0}$ independe da presença dos centros $E_{1}$, ou seja, eles não são precursores dos primeiros. Logo, acredita-se que os precursores dos centros $\left[\mathrm{E}_{1}{ }_{1}-\mathrm{Ge}\right]^{0}$ sejam os mesmos dos $\mathrm{E}_{1}{ }_{1}$ : provavelmente defeitos diamagnéticos constituídos por dois elétrons aprisionados em uma vacância de oxigênio, aqui representados por $\left[\mathrm{O}_{3} \mathrm{Si}\right]^{0}$ (Weil 1984). No momento, o que não pode ser esclarecido é a natureza dos defeitos que hospedam os íons $\mathrm{Li}^{+}$, quando as amostras são expostas a doses superiores a $8 \mathrm{kGy}$. Defeitos de estrutura ainda mais complexa, envolvendo íons $\mathrm{Li}^{+}$, têm sido propostos para explicar os sinais de espectros RPE obtidos a temperaturas criogênicas de quartzo irradiado com doses da ordem de $30 \mathrm{kGy}$ (Lees et al. 2003).

Uma análise detalhada da concentração de impurezas da Tab. 1 revela que nem todos os centros $\mathrm{Al}^{3+}$ são compensados pelo $\mathrm{Li}^{+}$, uma vez que as razões entre as concentrações de $\mathrm{Li}$ e $\mathrm{Al}$ ( $\mathrm{Li} / \mathrm{Al})$ são inferiores a 1. Outro compensador de carga relevante para os centros de $\mathrm{Al}$ em quartzo não irradiado são os prótons $\mathrm{H}^{+}$(Kats 1962, Bahadur 1989, Guzzo et al. 1997). Acredita-se que as medidas IV deste trabalho foram afetadas pela grande quantidade de fraturas das amostras, dificultando-se a estimativa da intensidade da banda associada ao defeito $\left[\mathrm{AlO}_{4} / \mathrm{H}\right]^{0}$. Os resultados da Tab. 1 também ajudam a explicar a saturação da banda associada ao defeito $\left[\mathrm{AlO}_{4}\right]^{0}$ no quartzo leitoso para doses acima de 48 kGy. Além da influência das fraturas, a menor razão entre as concentrações de Li e Al para esta variedade sugere menor quantidade de centros $\left[\mathrm{AlO}_{4} / \mathrm{Li}\right]^{0}$ tidos como precursores dos $\left[\mathrm{AlO}_{4}\right]^{0}$. Outra constatação decorrente das concentrações de impurezas é que não há centros $\left[\mathrm{GeO}_{4}\right]^{0}$ suficientes para abrigar os íons $\mathrm{Li}^{+}$removidos dos $\left[\mathrm{AlO}_{4} / \mathrm{Li}\right]^{0}$, ou seja, nestes cristais, a formação de centros $\left[\mathrm{GeO}_{4} / \mathrm{Li}\right]^{0}$ seria limitada pelo número de íons $\mathrm{Ge}^{4+}$. A possibilidade 
de haver outros defeitos relacionados aos íons $\mathrm{Li}^{+}$, como proposto por Lees et al. (2003) e Walsby et al. (2003), poderia explicar o destino desses íons após a irradiação.

Conforme mostrado no detalhe da Fig. 2A, a banda de absorção associada à coloração rósea $\mathrm{e}$ atribuída à transferência de carga entre íons $\mathrm{Fe}^{2+} \mathrm{e}$ $\mathrm{Ti}^{4+}$ foi observada em $495 \mathrm{~nm}$ para algumas amostras de coloração rósea acentuada. A análise dos espectros UV-Vis obtidos após irradiação revelou que esta banda não foi afetada pelas doses progressivas de radiação $\gamma$, tornou-se menos perceptível devido ao aparecimento da banda a $460 \mathrm{~nm}$ associada ao centro $\left[\mathrm{AlO}_{4}\right]^{0}$. Resultado semelhante foi observado por Cohen \& Makar (1984), quando quartzo róseo extraído de núcleos de pegmatito foi irradiado com raios X. Adicionalmente, com exceção da saturação da banda $\left[\mathrm{AlO}_{4}\right]^{0}$ para o quartzo leitoso, a Fig. 3 mostra que o quartzo do Pegmatito Taboa apresenta comportamento semelhante em função da dose de radiação $\gamma$, independentemente da intensidade de sua coloração natural. Essas constatações mostram que a natureza dos defeitos responsáveis pela coloração rósea do quartzo do Pegmatito Taboa é independente das impurezas estruturais de Al, Ge, Li e grupos OH. Portanto, baseado nesta análise e nos resultados preliminares de MEV, mostrados na Fig. 4, pode-se sugerir que a coloração rósea dos blocos de quartzo da PPB esteja relacionada à presença de inclusões submicrométricas de dumortierita.

A morfologia da fibra da Fig. 4 é semelhante àquelas observadas por Ma \& Rossman (2007). A ausência do boro nas análises de ICP-MS e MEVEDS do quartzo róseo pode ser explicada pela volatilização deste elemento durante a preparação da amostra. Naka \& Kurayasu (1991) e Karunasagar et al. (2000) relatam a volatilização do boro durante a digestão ácida (HF) da matriz de quartzo e propõem o uso de reagentes durante a preparação da amostra para contornar este problema. Por sua vez, Sah \& Brown (1997) relatam a perda do boro durante os processos de decomposição e evaporação da amostra, devido à alta volatilidade do $\mathrm{BF}_{3}$, que é oriundo dos ataques ácidos. Medidas adicionais em resíduos da digestão ácida estão previstas para elucidar a presença do boro e buscar evidências sobre a transferência de carga entre íons de $\mathrm{Fe}^{2+} \mathrm{e} \mathrm{Ti}^{4+}$, na estrutura da dumortierita no quartzo da PPB.

CONCLUSÕES Os resultados deste trabalho permitem concluir que a coloração do quartzo róseo-leitoso do Pegmatito Taboa da PPB é facilmente modificada por irradiação com raios $\gamma\left({ }^{60} \mathrm{Co}\right)$, tornando-se esfumaçada a partir de $2 \mathrm{kGy}$ e completamente escura para doses da ordem de $100 \mathrm{kGy}$. A suscetibilidade ao escurecimento deve-se à grande concentração de centros $\mathrm{Al}^{3+}$ compensados por $\mathrm{Li}^{+}$, $\left[\mathrm{AlO}_{4} / \mathrm{Li}\right]^{0}$, que, progressivamente, vão se transformando em centros $\left[\mathrm{AlO}_{4}\right]^{0}$. A mobilidade dos íons $\mathrm{Li}^{+}$, em função da dose de raios $\gamma$, mostrou que os centros E' ${ }_{1}$ não são os precursores dos $E^{\prime}{ }_{1}$ perturbados por Ge. O comportamento semelhante entre o quartzo de tonalidades rósea e leitosa em função da dose de radiação $\gamma$, a pequena diferença observada entre as concentrações de impurezas dessas amostras e o fato de terem sido identificadas fibras nanométricas apenas no resíduo da digestão ácida da amostra rósea permitem concluir que os mecanismos e os centros de defeitos, relacionados ao escurecimento dos cristais por radiação $\gamma$, são independentes da natureza da coloração rósea, cuja origem está provavelmente associada à presença de inclusões submicrométricas de dumortierita.

AGRADECIMENTOS Os autores agradecem às Professoras Helen J. Khoury (Departamento de Energia Nuclear da Universidade Federal de Pernambuco - UFPE) pela disponibilização da infraestrutura do Laboratório de Metrologia das Radiações Ionizantes e Maria Fernanda Pimentel (Departamento de Engenharia Química da UFPE) pela utilização do espectrômetro IV. As análises de microscopia eletrônica foram realizadas com o apoio técnico de Francisco Rangel do Centro de Tecnologias Estratégicas do Nordeste (Recife, PE). Este estudo contou com o apoio da Financiadora de Estudos e Projetos (FINEP), do Conselho Nacional de Desenvolvimento Científico e Tecnológico (CNPq) e do Programa Institucional de Bolsas de Iniciação Científica (PIBIC) do CNPq do CETEM/ MCT e da UFPE. 


\section{Referências}

Aines R.D. \& Rossman G.R. 1984. Water in minerals? A peak in the infrared. Journal of Geophysical Research, 89:4059-4071.

Applin K.R. \& Hicks B.D. 1987. Fibers of dumortierite in quartz. American Mineralogist, 72:170-172.

Bahadur H. 1989. Infrared characterization of natural and cultured quartz: the effect of electrodiffusion and irradiation. Journal of Applied Physics, 66:4973-4982.

Balitsky V.S., Makhina I.B., Prygov V.I., Mar'in A.A., Emel'chenko A.G., Fritsch E., et al. 1998. Russian synthetic pink quartz, Gems \& Gemology, 34(1):3443.

Barreto S.B., Guzzo P.L., Souza Neto J.A., Morais P.A.R., Carvalho Jr. A.B., Khoury H.J. 2009. Spectroscopic study of rose quartz from the Taboa Pegmatite (Borborema Province, Brazil) irradiated with high gamma doses. Estudos Geológicos, 19:52-56.

Beurlen H., Barreto S.B., Martin R., Melgarejo J., Da Silva M.R.R., Souza Neto J.A. 2009a. The Borborema Pegmatitic Province, NE-Brazil, revisted. . Estudos Geológicos, 19:62-66.

Beurlen H., Müller A., Silva D., Da Silva M.R.R. 2009b. Petrogenetic significance of trace-element concentrations in quartz from pegmatites of the Borborema Pegmatite Province, Northeastern Brazil. Estudos Geológicos, 19:72-76.

Cohen A.J. \& Makar L.N. 1984. Differing effects of ionizing radiation in massive and single crystal rose quartz. Neus Jahrbuch für Mineralogie - Monatshefte, 11:513-521.

Cox R.T. 1977. Optical absorption of the $\mathrm{d}^{4}$ ion $\mathrm{Fe}^{4+}$ in pleochroic amethyst quartz. Journal of Physics $C$ : Solid State Physics, 10(22):4631-4643.

Da Silva M.R.R., Höll R., Beurlen H. 1995. Borborema Pegmatitic Province: geological and geochemical characteristics. Journal of South American Earth Sciences, 8(3-4):355-364.

Goreva J.S., Ma C., Rossman G.R. 2001. Fibrous nanoinclusions in massive rose quartz: The origin of rose coloration. American Mineralogist, 86:466-472.

Götze J., Plötze M., Graupner T., Hallabauer D.K., Bray C.J. 2004. Trace element incorporation into quartz: A combined study by ICP-MS, electron spin resonance, cathodolumiescence, capillary ion analysis, and gas chromatography. Geochimica et Cosmochimica Acta, 68(18):3741-3759.

Guzzo P.L., Iwasaki F., Iwasaki H. 1997. Al-related centers in relation to $\gamma$-irradiation response in natural quartz. Physics and Chemistry of Minerals, 24(4):254-263.

Halliburton L.E., Koumvakalis N., Markes M.E., Martin J.J. 1981. Radiation effects in crystalline $\mathrm{SiO}_{2}$ : the role of aluminum. Journal of Applied Physics, 52(5):3565-3574.
Holden E.F. 1924. The cause of colour in rose quartz. American Mineralogist, 9:101-108.

Iwasaki H., Iwasaki F., Oliveira V.A.R., Hummel D.A.C., Pasquali M.A., Guzzo P.L., et al. 1991. Impurity content characterization of Brazilian natural quartz. Journal of Applied Physics, 30:1489-1495.

Jani M.G., Bossoli R.B., Halliburton L.E. 1983. Further characterization of the $\mathrm{E}^{\text {' }}$ center in crystalline $\mathrm{SiO}_{2}$. Physical Review B, 27(4):2285-2293.

Karunasagar D., Dash K., Chandrasekaran K., Arunachalam J. 2000. ICP-MS determination of trace amounts of boron in high-purity quartz. Atomic Spectrometry, 21:216-219.

Kats A. 1962. Hydrogen in $\alpha$-quartz. Philips Research Reports, 17:113-195, 201-279.

Kibar R., Garcia-Guinea J., Çetin A., Selvi S., Karan T., Cand N. 2007. Luminescent, optical and colour properties of natural rose quartz. Radiation Measurements, 42:1610-1617.

Koivula J.I. 1980. Citrine-amethyst quartz: Agemologically new material, Gems \& Gemology, 16(9):290-293.

Lameiras F.S., Nunes E.H.M., De Melo V.A.R., Liz O.R. 2006. Identificação de quartzos incolores para joalheria. Revista Escola de Minas, 59(1):129-133.

Larsen R.B., Henderson L., Ihlen P.M., Jacamon F. 2004. Distribution and petrogenetic behaviour of trace elements in granitc pegmatite quartz from south Norway. Contributions to Mineralogy and Petrology, 147:615-628.

Lees N.S., Walsby C.J., Willians J.A.S., Weil J.A., Claridge R.F.C. 2003. EPR of a hydrogen/double-lithium centre in $\alpha$-quartz. Physics and Chemistry of Minerals, 30(3):131-141.

Lehmann G., Moore W.J. 1966. Color center in amethyst quartz. Science, 152:1061-1062.

Lipson H.G. \& Kahan A. 1985. Infrared characterization and hydrogen defect centers in irradiated quartz. Journal of Applied Physics, 58:963-970.

Luz A.B., Lins F.A.F., Piquet B., Costa M.J., Coelho J.M. 2003. Pegmatitos do Nordeste: Diagnóstico sobre o aproveitamento racional e integrado. Série Rochas e Minerais Industrais, 9, Centro de Tecnologia Mineral, Rio de Janeiro, $97 \mathrm{p}$.

Ma C., Goreva J.S., Rossman G.R. 2002. Fibrous nanoinclusions in massive rose quartz: HRTEM and AEM investigations. American Mineralogist, 87:269276.

Ma C. \& Rossman G.R. 2007. Nano-mineralogy studies by advanced electron microscopy. Microscopy and Microanalysis, 13(Supplement 2):164-165.

Machmeyer D. \& Lehmann G. 1983. A trapped-hole center causing rose coloration of natural quartz. Zeitschrift für Kristallographie, 163:181-196. 
Mackey J.H. 1963. EPR study of impurity-related color centers in germanium-doped quartz. Journal of Chemical Physics, 39:74-83.

Martin J.J. 1984. Aluminum-related acoustic loss in AT-cut quartz crystals. Journal of Applied Physics, 56:25362540 .

Naka H. \& Kurayasu H. 1991. Determination of traceelements in high-purity quartz by inductively coupled plasma mass-spectrometry. Tetsu To Hagane-Jounal of the Iron and Steel Institute of Japan, 77:1959-1964.

Nuttal R.H.D. \& Weil J.A. 1981. The magnetic properties of the oxygen-hole aluminum centers in crystalline $\mathrm{SiO}_{2}$. I. $\left[\mathrm{AlO}_{4}\right]^{0}$. Canadian Journal of Physics, 59:16961708 .

1980. Two hydrogenic trapped-hole species in $\alpha$-quartz. Solid State Communications, 33:99-102.

O'Brien M.C.M. 1955. The structure of colour centres in smoky quartz. Proceedings of the Royal Society, A231:404-414.

Rossman G.R. 1994. Colored varieties of the silica minerals. Review in Mineralogy, 29: Silica, Eds. P.J. Heaney, C.T. Prewitt, G.V. Gibbs, p. 433-467.
Sah R.N. \& Brown P.H. 1997. Boron determination: A review of analytical methods. Microchemical Journal, 56:285-304.

Smith G., Vance E.R., Hasan Z., Edgar A., Runcman W.A. 1978. A charge transfer mechanism for the colour of rose quartz. Physica Status Solidi (A), 46:K135-K140.

Walsby C.J., Lees N.S., Claridge R.F.C., Weil J.A. 2003. The magnetic properties of oxygen-hole aluminum centres in crystalline $\mathrm{SiO} 2$. VI: A stable $\mathrm{AlO}_{4} / \mathrm{Li}$ centre. Canadian Journal of Physics, 81:583-598.

Weil J.A. 1984. A review of electron spin spectroscopy and its application to the study of paramagnetic defects in crystalline quartz. Physics and Chemistry of Minerals, 10:149-165.

Zichy V.J.I. 1972. Quantitative infrared analysis of polymeric material. In: Laboratory Methods in Infrared Spectroscopy. (eds.). R.G.J. Miller, B.C. Stage; Heyden \& Son: Londres, p. 48-70.

Manuscrito ID 22797

Recebido em: 28/08/2011

Aprovado em: 07/03/2012 